\title{
A six-step Block Unification Integrator for Numerical Solution of Fourth Order Boundary Value Problems
}

\author{
Mark I. Modebei ${ }^{\text {} l}$ and Raphael B. Adeniyi ${ }^{2}$ \\ 1,2 Department of Mathematics, University of Ilorin, P.M.B 1515, Ilorin, Nigeria. \\ ${ }^{1}$ gmarc . ify@gmail.com
}

\begin{abstract}
In this paper, a new 7th order continuous finite difference methods is proposed. These methods are derived using the Chebyshev polynomials as basis functions. The collocation approach is employed to obtain the main methods and additional methods used for solving general nonlinear fourth order two and four-points boundary value problems. Several numerical examples are shown to illustrate the strength of the method. To show the robustness of this method for high accuracy, we applied the method of line to discretize PDEs into system of fourth order ODEs and thus use the derived method to obtain approximate solution for the PDEs. The approximate solution obtained using the proposed methods is compared to the exact solutions of the problem, and other methods from existing literature. The Convergence of these methods is also guaranteed.
\end{abstract}

Keywords: Block Methods; Convergence; Finite Difference; Linear multistep. 2010 MSC No: 65L05, 65L06, 65L10, 65L12.

\section{Introduction}

In this work, the numerical approximation for the $\mu$ th-order problems of the type:

$$
\left.\begin{array}{c}
y^{(i v)}=f\left(x, y, y^{\prime}, \ldots, y^{\prime \prime \prime \prime}\right), \quad a \leq x \leq b \\
y(a)=\alpha_{0}, \quad y\left(c_{0}\right)=\gamma_{0} \\
y^{\prime}\left(c_{1}\right)=\gamma_{1}, \quad y^{\prime}(b)=\beta_{1}
\end{array}\right\}
$$

where $a, b, \alpha_{i}, \beta_{i}$ and $\gamma_{i}, i=0,1,2$ are real real numbers and $c_{0}, c_{1} \in(a, b)$ Other forms of boundary conditions considered are $y(a)=\alpha_{0}, \quad y\left(c_{0}\right)=\gamma_{0}, \quad y\left(c_{1}\right)=\gamma_{1}, \quad y(b)=\beta_{1}$ and $y(a)=\alpha_{0}, \quad y^{\prime}\left(c_{0}\right)=\gamma_{0}, \quad y^{\prime \prime}\left(c_{1}\right)=\gamma_{1}, \quad y^{\prime \prime \prime}(b)=$ $\beta_{1}$.

Problems arising from engineering and other sciences are modeled into linear and nonlinear of at least two points boundary value problems. Modeled problems with more that two points boundary conditions are referred to as multi-point boundary value problems (MBVPs). This kind of problem arise in different applied mathematics and physics. For example, MBVP models the flow of fluid such as water, oil and gas through ground layers, where each layer constitutes a subdomain, see [29]. The vibrations of a guy wire of a uniform cross-section and composed of $N$ parts of different densities, see 12 . Problems in the theory of elastic stability can be modeled by multi-point problems, large size bridges are sometimes constructed with multi-point supports which correspond to a multi-point boundary value condition, see [8, 9, 10].

\footnotetext{
${ }^{*}$ Corresponding author. Mark I. Modebei ${ }^{1}$ gmarc.ify@gmail.com
} 
So many numerical methods have been developed for the solutions of fourth order BVPs include Optimal Homotopy Asymptotic Method (OHAM) see [6], Quasi-Newtons and reproducing kernel method, see [29. Siddiqi and Akram [26, 27] used the Quintic Spline Solutions for Fourth Order Boundary-Value Problems. The Adomian decomposition method for solving multipoint boundary value problems, was also used in [28, Runge-Kutta Type Methods for Directly Solving Special Fourth-Order Ordinary Differential Equations was also used in [14, 16]. Readers may also see [3, [4, [7] and [22, just to mention few.

we derive a continuous linear multistep method (LMM) via a block unification technique, which is used to formulate a six-step block unification integrator (SBUI) using Chebyshev polynomials as basis functions with the collocation approach. Continuous LMM is derived and additional methods which are assembled and solved sequentially to obtain approximations $y_{n}$, to the solution of (1) directly at the points $\{x\}_{i}$.

The paper is organized as follows. In Section 2, we derive an approximation $Y(x)$ for the exact solution $y(x)$ which is continuous. Further more we state the specification of the methods and how the SBUI is obtained. In section 3 we discussed the order, local truncation error and convergence of the method and in section 4, we give some numerical examples to show the accuracy and reliability of the method. In the last section, we discuss the conclusion.

\section{Derivation of the Methods}

Here, the exact solution $y(x)$ is approximated by seeking the continuous method $Y(x)$ of the form

$$
Y(x)=\sum_{i=0}^{r+s-1} \rho_{i} T_{i}(x)
$$

with the fourth derivative given by

$$
Y^{(i v)}(x)=\sum_{i=0}^{r+s-1} \rho_{i} T_{i}^{(i v)}(x)
$$

where $x \in[a, b], \rho_{i}$ 's are coefficient to be determined, and $T_{i}(x)$ are Chebyshev polynomial basis function of degree $r+s-1, r$ is the number of interpolation points that satisfies $4<r \leq k$ and $s$ is the number of collocation points satisfying $0<s \leq k+1$. and $k$ indicates the number of steps and in this case, $k \geq 4$. We thus impose the following conditions as follows;

$$
\begin{aligned}
& Y\left(x_{n+i}\right)=y_{n+i}, \quad i=0,1,2, \ldots, r-1 \\
& Y^{(i v)}(x)=D Y\left(x_{n+i}\right)=f_{n+i}, \quad i=0,1,2, \ldots, s-1
\end{aligned}
$$

where $D=\frac{d^{4}}{d x^{4}}$

Interpolating (3) at $x_{n+i} ; i=0,1,2, \ldots, r-1$ and collocating (4) at $x_{n+s} ; s=0,1,2, \ldots, k$. which leads to the following systems of equations

$$
\begin{aligned}
\sum_{i=0}^{r+s-1} \rho_{i} T_{i}(x) & =y_{n+i} \\
\sum_{i=0}^{r+s-1} \rho_{i} T_{i}^{(i v)}(x) & =f_{n+i}
\end{aligned}
$$

where $\rho_{i}^{\prime} s$ are coefficients of the Chebyshev series. Thus we define the following interpolation and collocation in a 
single matrix as follows

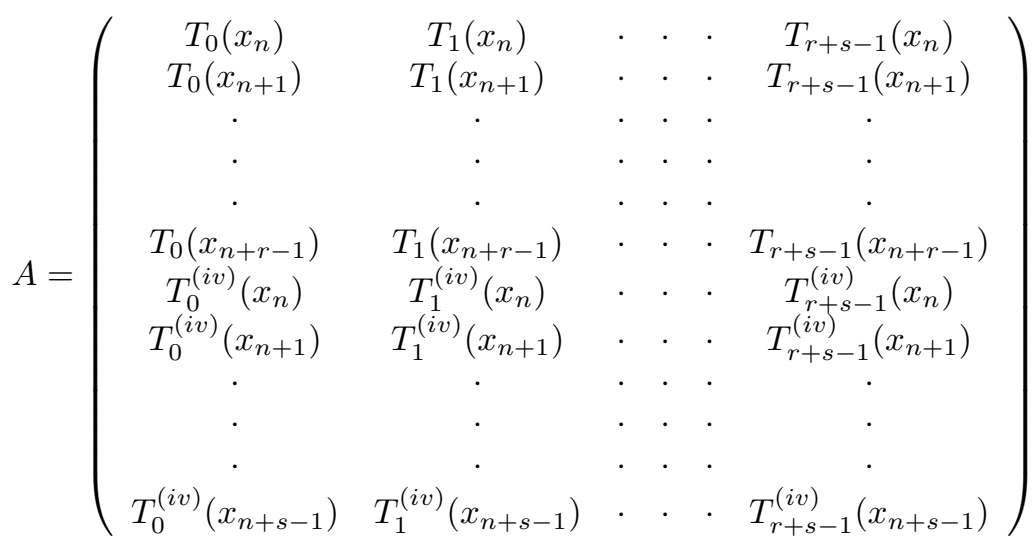

$$
\begin{aligned}
& B=\left(\begin{array}{llllll}
\rho_{0} & \rho_{1} & \cdot & \cdot & \cdot & \rho_{r+s-1}
\end{array}\right)^{T} \\
& C=\left(\begin{array}{llllllllllll}
y_{n} & y_{n+1} & \cdot & \cdot & y_{n+r-1} & f_{n} & f_{n} & \cdot & \cdot & f_{n+s-1}
\end{array}\right)^{T} \\
& E=\left(\begin{array}{lllll}
T_{0}(x) & T_{1}(x) & \cdot & T_{r+s-1}(x)
\end{array}\right)^{T}
\end{aligned}
$$

Then the system (7) and (8) is thus

$$
A \cdot B=C
$$

Hence we state the following theorem

Theorem 2.1. Let

$$
\begin{aligned}
& Y\left(x_{n+i}\right)=y_{n+i}, \quad i=0,1,2, \ldots, r-1 \\
& Y^{(i v)}(x)=D Y\left(x_{n+i}\right)=f_{n+i}, \quad i=0,1,2, \ldots, s-1
\end{aligned}
$$

be satisfied, the continuous $k$-step LMM (17) is derived from the equation

$$
Y(x)=C^{T}\left(A^{-1}\right)^{T} E
$$

Proof. Given the continuous scheme

$$
Y(x)=\sum_{i=0}^{r+s-1} \rho_{i} T_{i}(x)
$$

where $x \in[a, b], \rho_{i}$ 's are unknown coefficients, $T_{i}(x)$ 's are the Chebyshev polynomial basis functions of degree $r+s-1$. We can clearly write 13 as

$$
Y(x)=\rho_{0} T_{0}(x)+\rho_{1} T_{1}(x)+\rho_{2} T_{2}(x)+\cdots+\rho_{r+s-2} T_{r+s-2}(x)+\rho_{r+s-1} T_{r+s-1}(x)
$$

Then 14 can be written compactly in vector form as

$$
Y(x)=B^{T} E
$$

From $(9)$, by left inverse cancelation law we have

$$
B=A^{-1} C
$$

Hence, by 15 we have

$$
Y(x)=C^{T}\left(A^{-1}\right)^{T} E
$$

as required.

The equation (14) takes the form 


$$
Y(x)=\sum_{i=0}^{r-1} \alpha_{i}(t) h^{i} y_{n}^{(i)}+h^{4} \sum_{i=0}^{s-1} \beta_{i}(t) f_{n+i}
$$

where $\alpha_{i}(t)$ and $\beta_{i}(t)$ are continuous coefficients. The solution of $(1)$ is sought on the partition

$$
\pi N: a=x_{0}<x_{1}<x_{2}<\cdots<x_{n}<x_{n+1}<\cdots<x_{N}=b
$$

of the integration interval $[a, b]$ with a constant step size $h=\frac{b-a}{N}, N$ is the number of subinterval of integration.

It is note worthy that the continuous methods (17) and its derivatives $Y^{\prime}(x), Y^{\prime \prime}(x)$ and $Y^{\prime \prime \prime}(x)$ will be used to produce the main and additional methods which gives a total of 24 equations and are combined to provide all approximations on the entire interval for boundary value problems as stated in (1).

Applying the theorem (2.1), with $k=6, r=4, s=7$, and obtain the continuous coefficients $\alpha_{i}(x), \beta_{i}(x)$, expressed as functions of $t$ (whose expressions are not included), where $t h=x-x_{k-1}$. By evaluating (17) at $x=x_{n+i} i=1(1) 6$, the following 6-step LMM are obtained;

$$
\begin{aligned}
y_{n+1}= & y_{n}+\frac{3 h y_{n}^{\prime}}{2}+\frac{9}{8} h^{2} y_{n}^{\prime \prime}+\frac{9}{16} h^{3} y_{n}^{\prime \prime \prime}-h^{4}\left(\frac{95929 f_{n}}{3628800}-\frac{4001 f_{n+1}}{129600}+\frac{101729 f_{n+2}}{725760}\right. \\
& \left.-\frac{811 f_{n+3}}{30240}+\frac{10693 f_{n+4}}{725760}-\frac{4219 f_{n+5}}{907200}-\frac{2323 f_{n+6}}{3628800}\right) \\
y_{n+2}= & y_{n}+3 h y_{n}^{\prime}+\frac{9}{2} h^{2} y_{n}^{\prime \prime}+\frac{9}{2} h^{3} y_{n}^{\prime \prime \prime}+h^{4}\left(\frac{4127 f_{n}}{14175}+\frac{8782 f_{n+1}}{14175}-\frac{6425 f_{n+2}}{9072}\right. \\
& \left.+\frac{388 f_{n+3}}{945}-\frac{127 f_{n+4}}{567}+\frac{998 f_{n+5}}{14175}-\frac{137 f_{n+6}}{14175}\right) \\
y_{n+3}= & y_{n}+\frac{9 h y_{n}^{\prime}}{2}+\frac{81}{8} h^{2} y_{n}^{\prime \prime}+\frac{243}{16} h^{3} y_{n}^{\prime \prime \prime}+h^{4}\left(\frac{49239 f_{n}}{44800}+\frac{34263 f_{n+1}}{11200}-\frac{56129 f_{n+2}}{26880}\right. \\
& \left.+\frac{261 f_{n+3}}{160}-\frac{8019 f_{n+4}}{8960}+\frac{3159 f_{n+5}}{11200}-\frac{1737 f_{n+6}}{44800}\right) \\
y_{n+4}= & y_{n}+4 h y_{n}^{\prime}+8 h^{2} y_{n}^{\prime \prime}+\frac{32}{3} h^{3} y_{n}^{\prime \prime \prime}+h^{4}\left(\frac{628358656 f_{n}}{837019575}+\frac{543924224 f_{n+1}}{279006525}-\frac{114992431 f_{n+2}}{74401740}\right. \\
& \left.+\frac{184795136 f_{n+3}}{167403915}-\frac{33762304 f_{n+4}}{55801305}+\frac{843776 f_{n+5}}{4428675}-\frac{21911552 f_{n+6}}{837019575}\right) \\
y_{n+5}= & y_{n}+5 h y_{n}^{\prime}+\frac{25}{2} h^{2} y_{n}^{\prime \prime}+\frac{125}{6} h^{3} y_{n}^{\prime \prime \prime}+h^{4}\left(\frac{51613375 f_{n}}{33480783}+\frac{803750 f_{n+1}}{177147}-\frac{480390557 f_{n+2}}{178564176}\right. \\
& \left.+\frac{77537500 f_{n+3}}{33480783}-\frac{4709375 f_{n+4}}{3720087}+\frac{4453250 f_{n+5}}{11160261}-\frac{1836875 f_{n+6}}{33480783}\right) \\
& y_{n}+6 h y_{n}^{\prime}+18 h^{2} y_{n}^{\prime \prime}+36 h^{3} y_{n}^{\prime \prime \prime}+h^{4}\left(\frac{7808 f_{n}}{2835}+\frac{124928 f_{n+1}}{14175}-\frac{90733 f_{n+2}}{22680}\right. \\
& \left.+\frac{4096 f_{n+3}}{945}-\frac{928 f_{n+4}}{405}+\frac{2048 f_{n+5}}{2835}-\frac{1408 f_{n+6}}{14175}\right) \\
y_{n+6} &
\end{aligned}
$$

Evaluating $Y^{\prime}(x)$ of 17 at the points $x=x_{n+i} i=1(1) 6$, we obtain the first derivative additional method as;

$$
\begin{aligned}
y_{n+1}^{\prime}= & y_{n}^{\prime}+h y_{n}^{\prime \prime}+\frac{1}{2} h^{2} y_{n}^{\prime \prime \prime}+h^{3}\left(\frac{18685714 f_{n}}{837019575}+\frac{6227992 f_{n+1}}{279006525}-\frac{17182393 f_{n+2}}{178564176}\right. \\
& \left.+\frac{97504 f_{n+3}}{4782969}-\frac{627026 f_{n+4}}{55801305}+\frac{991432 f_{n+5}}{279006525}-\frac{409901 f_{n+6}}{837019575}\right) \\
y_{n+2}^{\prime}= & y_{n}^{\prime}+2 h y_{n}^{\prime \prime}+2 h^{2} y_{n}^{\prime \prime \prime}+h^{3}\left(\frac{106516688 f_{n}}{837019575}+\frac{67716224 f_{n+1}}{279006525}-\frac{50951657 f_{n+2}}{178564176}\right. \\
& \left.+\frac{29563648 f_{n+3}}{167403915}-\frac{5369008 f_{n+4}}{55801305}+\frac{8434304 f_{n+5}}{279006525}-\frac{496096 f_{n+6}}{119574225}\right) \\
y_{n+3}^{\prime}= & y_{n}^{\prime}+3 h y_{n}^{\prime \prime}+\frac{9}{2} h^{2} y_{n}^{\prime \prime \prime}+h^{3}\left(\frac{13774 f_{n}}{42525}+\frac{11992 f_{n+1}}{14175}-\frac{5875 f_{n+2}}{9072}+\frac{832 f_{n+3}}{1701}\right. \\
& \left.-\frac{758 f_{n+4}}{2835}+\frac{1192 f_{n+5}}{14175}-\frac{491 f_{n+6}}{42525}\right) \\
y_{n+4}^{\prime}= & y_{n}^{\prime}+4 h y_{n}^{\prime \prime}+8 h^{2} y_{n}^{\prime \prime \prime}+h^{3}\left(\frac{510727936 f_{n}}{837019575}+\frac{526440448 f_{n+1}}{279006525}-\frac{904408973 f_{n+2}}{892820880}\right. \\
& \left.+\frac{153915392 f_{n+3}}{167403915}-\frac{28274432 f_{n+4}}{55801305}+\frac{6375424 f_{n+5}}{39858075}-\frac{18420224 f_{n+6}}{837019575}\right) \\
y_{n+5}^{\prime}= & y_{n}^{\prime}+5 h y_{n}^{\prime \prime}+\frac{25}{2} h^{2} y_{n}^{\prime \prime \prime}+h^{3}\left(\frac{33034250 f_{n}}{33480783}+\frac{5357000 f_{n+1}}{1594323}-\frac{159598651 f_{n+2}}{127545840}\right. \\
& \left.+\frac{5198000 f_{n+3}}{33480783}-\frac{9271250 f_{n+4}}{11160261}+\frac{2921000 f_{n+5}}{11160261}-\frac{1205125 f_{n+6}}{33480783}\right) \\
y_{n}^{\prime} & +6 h y_{n}^{\prime \prime}+18 h^{2} y_{n}^{\prime \prime \prime}+h^{3}\left(\frac{61808 f_{n}}{42525}+\frac{74624 f_{n+1}}{14175}-\frac{12227 f_{n+2}}{9072}+\frac{4352 f_{n+3}}{1701}\right. \\
& \left.-\frac{496 f_{n+4}}{405}+\frac{5504 f_{n+5}}{14175}-\frac{2272 f_{n+6}}{42525}\right)
\end{aligned}
$$

Similarly, evaluating $Y^{\prime \prime}(x)$ of (17) at the points $x=x_{n+i} i=1(1) 6$, we obtain the second derivative additional method as; 


$$
\begin{aligned}
y_{n+1}^{\prime \prime}= & y_{n}^{\prime \prime}+h y_{n}^{\prime \prime \prime}+h^{2}\left(\frac{1093814 f_{n}}{18600435}+\frac{55528 f_{n+1}}{688905}-\frac{509932 f_{n+2}}{6200145}+\frac{184016 f_{n+3}}{2657205}\right. \\
& \left.-\frac{15698 f_{n+4}}{413343}+\frac{74248 f_{n+5}}{6200145}-\frac{6128 f_{n+6}}{3720087}\right) \\
y_{n+2}^{\prime \prime}= & y_{n}^{\prime \prime}+2 h y_{n}^{\prime \prime \prime}+h^{2}\left(\frac{563008 f_{n}}{3720087}+\frac{487040 f_{n+1}}{1240029}-\frac{612704 f_{n+2}}{2066715}+\frac{908032 f_{n+3}}{3720087}\right. \\
& \left.-\frac{821728 f_{n+4}}{6200145}+\frac{85888 f_{n+5}}{2066715}-\frac{15136 f_{n+6}}{2657205}\right) \\
y_{n+3}^{\prime \prime}= & y_{n}^{\prime \prime}+3 h y_{n}^{\prime \prime \prime}+h^{2}\left(\frac{2054 f_{n}}{8505}+\frac{776 f_{n+1}}{945}-\frac{32 f_{n+2}}{81}+\frac{3152 f_{n+3}}{8505}-\frac{194 f_{n+4}}{945}+\frac{184 f_{n+5}}{2835}-\frac{76 f_{n+6}}{8505}\right) \\
y_{n+4}^{\prime \prime}= & y_{n}^{\prime \prime}+4 h y_{n}^{\prime \prime \prime}+h^{2}\left(\frac{6161024 f_{n}}{18600435}+\frac{520192 f_{n+1}}{413343}-\frac{1939456 f_{n+2}}{6200145}+\frac{9396224 f_{n+3}}{18600435}-\frac{21248 f_{n+4}}{76545}\right. \\
& \left.+\frac{77824 f_{n+5}}{885735}-\frac{45056 f_{n+6}}{3720087}\right) \\
y_{n+5}^{\prime \prime}= & y_{n}^{\prime \prime}+5 h y_{n}^{(3)}+h^{2}\left(\frac{1568950 f_{n}}{3720087}+\frac{299000 f_{n+1}}{177147}-\frac{2500 f_{n+2}}{15309}+\frac{2950000 f_{n+3}}{3720087}-\frac{458750 f_{n+4}}{1240029}\right. \\
& \left.+\frac{47800 f_{n+5}}{413343}-\frac{59000 f_{n+6}}{3720087}\right) \\
y_{n+6}^{\prime \prime}= & y_{n}^{\prime \prime}+6 h y_{n}^{\prime \prime \prime}+h^{2}\left(\frac{4352 f_{n}}{8505}+\frac{6016 f_{n+1}}{2835}-\frac{32 f_{n+2}}{945}+\frac{10496 f_{n+3}}{8505}-\frac{32 f_{n+4}}{81}+\frac{128 f_{n+5}}{945}-\frac{32 f_{n+6}}{1701}\right)
\end{aligned}
$$

Lastly, evaluating $Y^{\prime \prime \prime}(x)$ of 17 at the points $x=x_{n+i} i=1(1) 6$, we obtain the third derivative additional method

$$
\begin{aligned}
y_{n+1}^{\prime \prime \prime}= & y_{n}^{\prime \prime \prime}+h\left(\frac{1656502 f_{n}}{18600435}+\frac{1251248 f_{n+1}}{6200145}-\frac{383918 f_{n+2}}{2066715}+\frac{2847328 f_{n+3}}{18600435}-\frac{515594 f_{n+4}}{6200145}+\frac{17968 f_{n+5}}{688905}\right. \\
& \left.-\frac{66506 f_{n+6}}{18600435}\right) \\
y_{n+2}^{\prime \prime \prime}= & y_{n}^{\prime \prime \prime}+h\left(\frac{341872 f_{n}}{3720087}+\frac{163712 f_{n+1}}{413343}-\frac{1180288 f_{n+2}}{6200145}+\frac{3013376 f_{n+3}}{18600435}-\frac{183056 f_{n+4}}{2066715}+\frac{172672 f_{n+5}}{6200145}\right. \\
& \left.-\frac{71104 f_{n+6}}{18600435}\right) \\
y_{n+3}^{\prime \prime \prime}= & y_{n}^{\prime \prime \prime}+h\left(\frac{2278 f_{n}}{25515}+\frac{752 f_{n+1}}{1701}+\frac{22 f_{n+2}}{8505}+\frac{2656 f_{n+3}}{25515}-\frac{538 f_{n+4}}{8505}+\frac{176 f_{n+5}}{8505}-\frac{74 f_{n+6}}{25515}\right) \\
y_{n+4}^{\prime \prime \prime}= & y_{n}^{\prime \prime \prime}+h\left(\frac{1680256 f_{n}}{18600435}+\frac{2673152 f_{n+1}}{6200145}+\frac{10624 f_{n+2}}{76545}+\frac{3607552 f_{n+3}}{18600435}-\frac{539456 f_{n+4}}{6200145}+\frac{54784 f_{n+5}}{2066715}\right. \\
& \left.-\frac{66944 f_{n+6}}{18600435}\right) \\
y_{n+5}^{\prime \prime \prime}= & y_{n}^{\prime \prime \prime}+h\left(\frac{336190 f_{n}}{3720087}+\frac{178000 f_{n+1}}{413343}+\frac{178750 f_{n+2}}{1240029}+\frac{1420000 f_{n+3}}{3720087}-\frac{1250 f_{n+4}}{15309}+\frac{32240 f_{n+5}}{1240029}\right. \\
& \left.-\frac{13250 f_{n+6}}{3720087}\right) \\
y_{n+6}^{\prime \prime \prime}= & y_{n}^{\prime \prime \prime}+h\left(\frac{2288 f_{n}}{25515}+\frac{3712 f_{n+1}}{8505}+\frac{1024 f_{n+2}}{8505}+\frac{12032 f_{n+3}}{25515}+\frac{464 f_{n+4}}{8505}+\frac{128 f_{n+5}}{8505}-\frac{64 f_{n+6}}{25515}\right)
\end{aligned}
$$

The main method (18) and the additional methods 19-21) together for the block unification integrator that will be assembled to form a single block and would be used in sequence over the interval $\left[x_{n}, x_{n+6}\right], n=0,6, N-6, N$ is the number of subinterval which must be a multiple of 6 .

\section{Order and Truncation Error And Convergence}

\subsection{Local truncation error}

The linear differential operator $\mathcal{L}[z(x) ; h]$ defined by

$$
\mathcal{L}[z(x) ; h]=z(x+i h)-\sum_{i=0}^{3} \alpha_{i} h^{i} z(x+i h)-h^{4} \sum_{i=0}^{6} \beta_{i} z^{(i v)}(x+i h)
$$

Expanding 22 in Taylor series, we obtain

$$
\mathcal{L}[y(x) ; h]=C_{0} z(x)+C_{1} h z^{\prime}(x)+C_{2} h^{2} z^{\prime \prime}(x)+\cdots+C_{p} h^{q} z^{(p)}(x)+O\left(h^{(p+1)}\right)
$$

where $C_{j}$ are constants such that

$$
C_{0}=C_{1}=C_{2}=\cdots=C_{p+3}=0, C_{p+4} \neq 0
$$


so that

$$
\mathcal{L}[z(x) ; h]=C_{p+4} h^{p+4} z^{(p+4)}(x)+O\left(h^{(p+5)}\right)
$$

In this case, $C_{p+4}$ is the error constant (see Lambert [15]). A linear difference operator $\mathcal{L}[z(x) ; h]$ is said to be consistent of order $p$ if

$$
\mathcal{L}[z(x) ; h]=O\left(h^{(p+4)}\right)
$$

The error constants $C_{p+4}^{\tau_{j}^{(\mu)}}$ for $j=1(1) 6, \mu=0,1,2,3$ are given below.

$$
\begin{aligned}
& C_{p+4}^{\tau_{1,2,3,5,6}}=\left(-\frac{1}{3024},-\frac{1}{756},-\frac{1}{336},-\frac{229}{665280}, \frac{107}{997920}, \frac{1273}{9979200}\right)^{T} \\
& C_{p+, 3,4,5,6}^{\tau_{1}^{\prime}}=\left(-\frac{617}{3326400},-\frac{761}{1425600},-\frac{1427}{997920},-\frac{1549}{665280}, \frac{41}{14400},-\frac{41}{302400}\right)^{T} \\
& C_{p+, 4,4,5,6}^{\tau_{1 \prime 2}^{\prime \prime}}=\left(\frac{1}{43200},-\frac{1}{3024},-\frac{23}{33600},-\frac{53}{100800},-\frac{1061}{302400},-\frac{8737}{907200}\right)^{T} \\
& C_{p+4}^{\tau_{1,2,3,4,5,6}^{\prime \prime \prime}}=\left(\frac{3151}{1814400},-\frac{151}{129600}, \frac{751}{1814400}, \frac{151}{129600}, \frac{3151}{1814400},-\frac{8737}{907200}\right)^{T}
\end{aligned}
$$

In what follows, the convergence of the method is established in theorem:

Theorem 3.1 ([18]). Let $\bar{Y}$ be the approximate solution vector of the exact solution $Y$ for the system formed by combining the methods (18) through (21). Let $e_{i}=\left|y\left(x_{i}\right)-y_{i}\right|, e_{i}^{\prime}=\left|y^{\prime}\left(x_{i}\right)-y_{i}^{\prime}\right|, e_{i}^{\prime \prime}=\left|y^{\prime \prime}\left(x_{i}\right)-y_{i}^{\prime \prime}\right|, e_{i}^{\prime \prime \prime}=\left|y^{\prime \prime \prime}\left(x_{i}\right)-y_{i}^{\prime \prime \prime}\right|$ and $e_{i}^{(i v)}=\left|y^{(i v)}\left(x_{i}\right)-y_{i}^{(i v)}\right|$ be defined as the error for $i=1, \ldots, N$ where the exact solution $y(x) \in C^{n}[a, b]$. Define $\|E\|_{\infty}=\|y-\bar{y}\|_{\infty}$, then the method is seventh-order convergent. That is $\|E\|_{\infty}=O\left(h^{7}\right)$.

Proof We write 18 through 21$]$ in the exact form

$$
A Y-h^{5} B F(Y)+\bar{C}+L(h)=0
$$

where $A$ is an $4 N \times 4 N$ coefficient matrix defined by

$$
A=\left[\begin{array}{ccc}
A_{11} & \cdots & A_{14} \\
\vdots & \ddots & \vdots \\
A_{41} & \cdots & A_{44}
\end{array}\right]
$$

$A_{i j}$ are $N \times N$ matrices for $i=1(1) 6, j=1$, and $A_{22}=A_{33}=A_{44}=A_{55}=I$, where $I$ is an $N \times N$ identity matrices and $A_{i j}=0$ for $i=1(1) 6 ; j=2(1) 6 ; i \neq j$.

Similarly, $B$ is a $4 N \times 4 N$ coefficient matrix defined by

$$
B=\left[\begin{array}{ccc}
B_{11} & \cdots & B_{14} \\
\vdots & \ddots & \vdots \\
B_{41} & \cdots & B_{44}
\end{array}\right]
$$

with $B_{i j}$ an $N \times N$ matrices for $i=1(1) 6, j=1$, and $B_{i j}$ are an $N \times$ zero matrix for $i=1(1) 6 ; j=2(1) 6$; and

$\bar{C}=\left(-h y_{0}^{\prime}-\frac{11 y_{0}}{6}-\frac{193 h f_{0}}{20160},-h^{2} y_{0}^{\prime \prime}+2 y_{0}+\frac{4463 h^{2} f_{0}}{60480},-h^{3} y_{0}^{\prime \prime \prime}-y_{0, m}-\frac{53 h^{3} f_{0}}{160},+y_{0}-\frac{h^{4} f_{0}}{720},-y_{0}+\frac{-31 h^{4} f_{0}}{60480}, \frac{1}{6} y_{0}+\frac{11 h^{4} f_{0}}{12096},-\frac{1}{3} y_{0}+\right.$ $\left.\frac{-23 h^{4} f_{0}}{20160}, \frac{11}{6} y_{0}-\frac{151 h^{4} f_{0}}{60480}, y_{0}+\frac{-157 h^{4} f_{0}}{60480}, \frac{11 h^{4} f_{0}}{60480},-y_{0}+\frac{-73 h^{4} f_{0}}{60480}, y_{0}-\frac{409 h^{4} f_{0}}{60480},-y_{0}+\frac{5 h^{4} f_{0}}{288},-y_{0}+\frac{-13 h^{4} f_{0}}{1440},-y_{0}+\frac{h^{4} f_{0}}{160}, y_{0}-\frac{h^{4} 29 f_{0}}{1440}, 0 \ldots, 0\right)$

$L(h)$ is the truncation error of the formulas 18 through $(21)$, defined as

$$
L(h)=\left(C^{\tau_{1}} \ldots, C^{\tau_{N}}, C^{\tau_{1}^{\prime}}, \ldots, C^{\tau_{N}^{\prime}}, C^{\tau_{1}^{\prime \prime}}, \ldots, C^{\tau_{N}^{\prime \prime}}, C^{\tau_{1}^{\prime \prime \prime}}, \ldots, C^{\tau_{N}^{\prime \prime \prime}}, C_{1}^{\tau^{(i v)}}, \ldots, C^{\tau_{N}^{(i v)}}\right)^{T}
$$

and

$$
\begin{gathered}
Y=\left(y\left(x_{1}\right), \ldots, y\left(x_{N}\right), y^{\prime}\left(x_{1}\right), \ldots, y^{\prime}\left(x_{N}\right), y^{\prime \prime}\left(x_{1}\right), \ldots, y^{\prime \prime}\left(x_{N}\right), y^{\prime \prime \prime}\left(x_{1}\right), y^{\prime \prime \prime}\left(x_{N}\right), y^{(i v)}\left(x_{1}\right), \ldots, y^{(i v)}\left(x_{N}\right)\right)^{T} \\
F(Y)=\left(f_{1}, \ldots, f_{N}, f_{1}^{\prime}, \ldots, f_{N}^{\prime}, f_{1}^{\prime \prime}, \ldots, f_{N}^{\prime \prime}, f_{1}^{\prime \prime \prime}, \ldots, f_{N}^{\prime \prime \prime}, f_{1}^{(i v)}, \ldots, f_{N}^{(i v)}\right)^{T}
\end{gathered}
$$

The approximate form of the system is given as

$$
A \bar{Y}-h^{5} B F(\bar{Y})+\bar{C}=0
$$

where $\bar{Y}$ is the approximate solution of the vector $Y$ Define the vector $E$ as

$$
E=\bar{Y}-Y=\left(e_{1}, \ldots, e_{N}, e_{1}^{\prime}, \ldots, e_{N}^{\prime}, e_{1}^{\prime \prime}, \ldots, e_{N}^{\prime \prime}, e_{1}^{\prime \prime \prime}, \ldots, e_{N}^{\prime \prime \prime}, e_{1}^{(i v)}, \ldots, e_{N}^{(i v)}\right)^{T}
$$


where

$$
\bar{Y}=\left(y_{1}, \ldots, y_{N}, y_{1}^{\prime}, \ldots, y_{N}^{\prime}, y_{1}^{\prime \prime}, \ldots, y_{N}^{\prime \prime}, y_{1}^{\prime \prime \prime}, \ldots, y_{N}^{\prime \prime \prime}, y_{1}^{(i v)}, \ldots, y_{N}^{(i v)}\right)^{T}
$$

Subtracting 26) from (27) and applying the mean value theorem, we obtain the error system as

$$
\left(A-B J_{f}\right) E=L(h)
$$

where $J_{f}$ is the Jacobian matrix

$$
J_{f}=\left(\begin{array}{ccc}
J_{11} & \cdots & J_{14} \\
\vdots & & \vdots \\
J_{41} & \cdots & J_{44}
\end{array}\right)
$$

whose entries $J_{i j}$ are $N \times N$ matrices given as $J_{11}=\left(\begin{array}{ccc}\frac{\partial f_{1}}{\partial y_{1}} & \cdots & \frac{\partial f_{1}}{\partial y_{N}} \\ \vdots & & \vdots \\ \frac{\partial f_{N}}{\partial y_{1}} & \cdots & \frac{\partial f_{N}}{\partial y_{N}}\end{array}\right)$;

$$
\begin{aligned}
J_{1, j+1} & =\left(\begin{array}{ccc}
\frac{\partial f_{1}}{\partial y_{1}^{(j)}} & \cdots & \frac{\partial f_{1}}{\partial y_{N}^{(j)}} \\
\vdots & & \vdots \\
\frac{\partial f_{N}}{\partial y_{1}^{(j)}} & \cdots & \frac{\partial f_{N}}{\partial y_{N}^{(j)}}
\end{array}\right) \text { for } j=1,2,3 \\
J_{i, j+1} & =h^{i}\left(\begin{array}{ccc}
\frac{\partial f_{1}^{(i-1)}}{\partial y_{1}^{(j)}} & \cdots & \frac{\partial f_{1}^{(i-1)}}{\partial y_{N}^{(j)}} \\
\vdots & & \vdots \\
\frac{\partial f_{N}^{(i-1)}}{\partial y_{1}^{(j)}} & \cdots & \frac{\partial f_{N}^{(i-1)}}{\partial y_{N}^{(j)}}
\end{array}\right) ; \text { for } j=0,1,2,3 \text {. and for } i=2, \ldots, 4 .
\end{aligned}
$$

Let $M=-B J_{f}$ be a matrix of dimension $4 N$ so that 28 becomes

$$
(A+M) E=L(h)
$$

and for sufficiently small $h, A+M$ is a monotone matrix and thus nonsingular (see [? ]). Hence

$$
\begin{aligned}
(A+M)^{-1} & =D=\left(d_{i j}\right) \geq 0 \\
\sum_{j=1}^{4 M} d_{i j} & =O\left(h^{-4}\right) \\
E & =D L(h) \\
\|E\| & =\|D L(h)\|=O\left(h^{-4}\right) O\left(h^{11}\right) \\
& =O\left(h^{7}\right)
\end{aligned}
$$

which shows that the method is 7th order convergent, that is, with a global error of order $O\left(h^{7}\right)$.

Implementation: Our main method (18) through 21] is implemented efficiently by combining them in a single matrix equation simultaneously of the form

$$
A_{0} V_{\mu}=A_{1} V_{\mu-1}+h^{4} B_{1} F_{\mu-1}+h^{4} B_{0} F_{\mu}, \quad \mu=1, \ldots, \Gamma, \quad n=0,4, \ldots, N-6
$$

where

$$
\begin{aligned}
& V_{\mu}=\left(y_{1}, \ldots, y_{n+6}, y_{1}^{\prime}, \ldots, y_{n+6}^{\prime}, y_{1}^{\prime \prime}, \ldots, y_{n+6}^{\prime \prime}, y_{1}^{\prime \prime \prime}, \ldots, y_{n+6}^{\prime \prime \prime}, y_{1}^{(i v)}, \ldots, y_{n+6}^{(i v)}\right)^{T} \\
& V_{\mu-1}=\left(y_{0}, \ldots, y_{n-5}, y_{0}^{\prime}, \ldots, y_{n-5}^{\prime}, y_{0}^{\prime \prime}, \ldots, y_{n-5}^{\prime \prime}, y_{0}^{\prime \prime \prime}, \ldots, y_{n-5}^{\prime \prime \prime}, y_{0}^{(i v)}, \ldots, y_{n-5}^{(i v)}\right)^{T} \\
& F_{\mu-1}=\left(f_{0}, \ldots, f_{n-5}, f_{0}^{\prime}, \ldots, f_{n-5}^{\prime}, f_{0}^{\prime \prime}, \ldots, f_{n-5}^{\prime \prime}, f_{0}^{\prime \prime \prime}, \ldots, f_{n-5}^{\prime \prime \prime}, f_{0}^{(i v)}, \ldots, f_{n-5}^{(i v)}\right)^{T} \\
& F_{\mu}=\left(f_{1}, \ldots, f_{n+6}, f_{1}^{\prime}, \ldots, f_{n+6}^{\prime}, f_{1}^{\prime \prime}, \ldots, f_{n+6}^{\prime \prime}, f_{1}^{\prime \prime \prime}, \ldots, f_{n+6}^{\prime \prime \prime}, f_{1}^{(i v)}, \ldots, f_{n+6}^{(i v)}\right)^{T}
\end{aligned}
$$

The positive integer $\Gamma=\frac{N}{6}$ is the number of blocks.

The Method has been implemented using the system Mathematica, enhanced by the feature NSolve[] for linear problems while nonlinear problems were solved by Newton's method enhanced by the feature FindRoot[], as summarized in the algorithm below. We begin by noting that the solution of the problem (1) is sought in the subinterval $\pi_{N}=\left\{a=x_{0}<x_{1}<\ldots<x_{N}=b\right\}$, where $h=\frac{b-a}{N}$ is a constant step-size of the partition of $\pi_{N}, N$ is the number of subintervals and $n$ the grid index.

Step 1: Use the block of (31) for $\mu=1, n=0$ to obtain $V_{1}$ on the rectangle $\left[y_{0}, y_{6}\right] \times[a, b]$, similarly, for $\mu=2, n=6$ so that $V_{2}$ is obtained on the rectangle $\left[y_{6}, y_{12}\right] \times[a, b]$, and on the rectangle $\left[y_{12}, y_{18}\right] \times[a, b], \ldots,\left[y_{N-6}, y_{N}\right] \times[a, b]$ for $\mu=3, \ldots \Gamma$, $n=12,18, \ldots, N-6$, we thus obtain $V_{3}, \ldots, V_{\Gamma}$.

Step 2: Solve the unified block given by the system $V_{1} \cup V_{2} \bigcup \ldots \cup V_{\Gamma}$, obtained in step 1.

Step 3:The solution of 11 is approximated by the solutions in step 2 as $y=\left[y\left(x_{1}\right), y\left(x_{2}\right), \ldots, y\left(x_{n}\right)\right]^{T}, n=1,2, \ldots, N$. 


\section{Numerical Examples}

In this part, we implement our derived method using numerical examples to show the high level of accuracy and efficiency of this method.

Problem 1. [6] Consider the following singular fourth order four-point boundary value problem

$$
\begin{aligned}
& x^{4}(1-x) y^{(i v)}(x)+300 e^{x / 2} y^{\prime \prime \prime}(x)+200 \sin (\sqrt{x}) y^{\prime \prime}(x)+2 y^{\prime}(x)+x y^{3}(x)=f(x), \\
& y(0)=0, \quad y(1 / 2)=\sin (1 / 2), \quad y(1 / 4)=\sin (1 / 4), \quad y(1)=\sin (1), \quad 0 \leq x \leq 1
\end{aligned}
$$

where

$f(x)=\left(e^{x}-1\right)^{2} \sin ^{2}(x)-2 \sin (\sqrt{x}) \sin (x)-e^{x / 2} \cos (x)+x \sin (\sin (x))+\cos (x) \sinh (x)$ The exact solution is given by $y(x)=$ $\sinh x$.

Table 1: error in Problem 1:

\begin{tabular}{lll}
\multicolumn{2}{r}{ methods in $[6]$} & SBUI \\
\hline method 1 & $1.8 \mathrm{E}-12$ & $6.44 \mathrm{E}-15$ \\
method 2 & $5.6 \mathrm{E}-13$ & \\
method 3 & $2.2 \mathrm{E}-14$ & \\
\end{tabular}

Problem 2. We consider the following nonlinear boundary value problem also found in [1].

$$
\begin{gathered}
y^{(i v)}(x)=(1+c) y^{\prime \prime}(x)-c y(x)+\frac{1}{2} c x^{2}-1, x \in[0,1] \\
y(0)=0, \quad y^{\prime}(0)=0, \quad y(1)=1.5+\sinh (1), \quad y^{\prime}(1)=1+\cos (1)
\end{gathered}
$$

with solution (independent of $c$ ) given as $y(x)=1+\frac{1}{2} x^{2}+\sinh (x)$

Table 2: error in Problem 2:

\begin{tabular}{rcc}
\hline $\mathrm{x}$ & SBUI & HBVP 1$]$ \\
\hline 0.1 & $4.58 \mathrm{E}-16$ & $5.44 \mathrm{E}-10$ \\
0.2 & $6.94 \mathrm{E}-16$ & $6.71 \mathrm{E}-10$ \\
0.3 & $1.67 \mathrm{E}-16$ & $9.72 \mathrm{E}-11$ \\
0.4 & $2.94 \mathrm{E}-15$ & $2.49 \mathrm{E}-10$ \\
0.5 & $3.66 \mathrm{E}-15$ & $1.85 \mathrm{E}-11$ \\
0.6 & $2.55 \mathrm{E}-15$ & $2.03 \mathrm{E}-10$ \\
0.7 & $3.89 \mathrm{E}-15$ & $1.65 \mathrm{E}-10$ \\
0.8 & $7.77 \mathrm{E}-16$ & $7.51 \mathrm{E}-10$ \\
0.9 & $4.44 \mathrm{E}-15$ & $5.90 \mathrm{E}-10$
\end{tabular}

Table 2 gives a clear comparison between SBUI and HBVP in [1] in problem 2. The errors obtained show that the SBUI is more accurate.

Problem 3. We consider the following nonlinear boundary value problem also found in [1].

$$
\begin{gathered}
y^{(i v)}(x)=y(x)+y^{\prime \prime}(x)+e^{x}(x-3), \quad x \in[0,1] \\
y(0)=0, \quad y^{\prime}(0)=0, \quad y(1)=0, \quad y^{\prime}(1)=-e
\end{gathered}
$$

with exact solution $e^{x}(1-x)$. 
Table 3: Maximum error in Problem 3:

\begin{tabular}{ccc}
\hline $\mathrm{x}$ & SBUI & HBVP 1 \\
\hline 0.1 & $9.99 \mathrm{E}-16$ & $1.97 \mathrm{E}-13$ \\
0.2 & $1.44 \mathrm{E}-16$ & $1.56 \mathrm{E}-13$ \\
0.3 & $1.09 \mathrm{E}-16$ & $1.83 \mathrm{E}-13$ \\
0.4 & $1.77 \mathrm{E}-14$ & $2.06 \mathrm{E}-13$ \\
0.5 & $2.10 \mathrm{E}-14$ & $2.02 \mathrm{E}-13$ \\
0.6 & $1.67 \mathrm{E}-14$ & $2.25 \mathrm{E}-13$ \\
0.7 & $1.05 \mathrm{E}-14$ & $2.04 \mathrm{E}-13$ \\
0.8 & $1.67 \mathrm{E}-15$ & $1.98 \mathrm{E}-13$ \\
0.9 & $3.33 \mathrm{E}-16$ & $2.18 \mathrm{E}-13$
\end{tabular}

Table 3 shows the comparison between SBUI and HBVP in [1] in problem 3. The errors obtained show that the SBUI is superior.

Problem 4. Consider the following nonlinear four points BVP, 29].

with exact solution $y(x)=x^{4}$.

$$
\begin{gathered}
y^{(i v)}(x)-y(x) y^{\prime}(x)=4 x^{7}+24, x \in[0,1] \\
y(0)=0, \quad y^{\prime \prime \prime}(0.25)=6, \quad y^{\prime \prime}(0.5)=3, \quad y(1)=1
\end{gathered}
$$

Table 4: Error of methods for Problem 4, $\mathrm{h}=0.25$ :

\begin{tabular}{rrrc}
\hline$x$ & $y_{a p p}$ & $y_{E x}$ & Er \\
\hline 0.1 & 0.0001 & 0.0001 & $5.21521 \times 10^{-17}$ \\
0.2 & 0.0016 & 0.0016 & $2.15154 \times 10^{-17}$ \\
0.3 & 0.0081 & 0.0081 & $3.54854 \times 10^{-16}$ \\
0.4 & 0.0256 & 0.0256 & $1.44854 \times 10^{-16}$ \\
0.5 & 0.0625 & 0.0625 & $6.52587 \times 10^{-16}$ \\
0.6 & 0.1296 & 0.1296 & $9.54454 \times 10^{-16}$ \\
0.7 & 0.2401 & 0.2401 & $1.74447 \times 10^{-16}$ \\
0.8 & 0.4096 & 0.4096 & $6.45451 \times 10^{-16}$ \\
0.9 & 0.6561 & 0.6561 & $1.57321 \times 10^{-16}$ \\
\hline
\end{tabular}

Table 5: error in Problem 4:

\begin{tabular}{ccccc}
\hline \multicolumn{1}{c}{ Method in [28 } & Method in [13] & Method in [29] & SBUI \\
\hline method 1: $2.2 \times 10^{-9}$ & $k_{1}=1: 3.4 \times 10^{-4}$ & method 1 & $1.8 \times 10^{-12}$ & $\mathrm{~N}=4: 8.1 \times 10^{-16}$ \\
method 2: $1.3 \times 10^{-9}$ & $k_{1}=2: 7.1 \times 10^{-7}$ & method 2 & $5.6 \times 10^{-13}$ & $\mathrm{~N}=8: 1.4 \times 10^{-17}$ \\
method 3: $1.0 \times 10^{-10}$ & $k_{1}=3: 5.4 \times 10^{-9}$ & method 3 & $2.2 \times 10^{-14}$ & $\mathrm{~N}=12: 2.1 \times 10^{-16}$ \\
\hline
\end{tabular}

In what follows, we consider forth order parabolic PDEs which we carried a semi-discretisation using the method of line as described in [11, 17, 25].

Problem 5. Consider the linear fourth-order parabolic equation, ([24].

$$
y_{t t}+y_{x x x x}=\left(\pi^{4}-1\right) \sin \pi x \cos t, \quad 0 \leq x \leq 1, \quad t \geq 0
$$

subject to the initial conditions

$$
y(x, 0)=\sin \pi x, \quad y_{t}(x, 0)=0, \quad 0 \leq x \leq 1
$$

and with appropriate boundary conditions

$$
y(0, t)=y(1, t)=y_{x x}(0, t)=y_{x x}(1, t)=0, \quad t \geq 0
$$

with the exact solution $y(x, t)=\sin \pi x \cos t$.

Here, following [23], the problem on discretizing the time variable, becomes

$$
\frac{y_{m+1}-2 y_{m}+y_{m-1}}{(\Delta t)^{2}}+\frac{d^{4} y_{m}}{d x^{4}}=g_{m}, \quad 0 \leq x \leq 1, \quad m=1, \ldots M-1
$$

where $\Delta t=\left(L_{4}-L_{3}\right) / M, t_{m}=L_{3}+m \Delta t, m=0,1, \ldots, M, y=\left[y_{1}(x), \ldots, y_{M}(x)\right]^{T}, y_{m}(x) \approx y\left(x, t_{m}\right), g=\left[g_{1}(x), \ldots, g_{m}(x)\right]^{T}$ and $g_{m}(x) \approx g\left(x, t_{m}\right)=\left(\pi^{4}-1\right) \sin \pi x \cos t_{m}$, which is expressed in the form

$$
y^{(4)}=f\left(x, y, y^{\prime}, y^{\prime \prime}, y^{\prime \prime \prime}\right)=A y+g
$$


$A$ is an $(M-1) \times(M-1)$ matrix arising from the discretized system, and $g$ is a vector of constants.

Problem 6. Consider the following homogenous fourth-order parabolic equation, 20].

$$
y_{t t}-y_{x x x x}=0, \quad 0 \leq x \leq 1, \quad t \geq 0
$$

subject to the initial conditions

$$
y(x, 0)=\sin \pi x, \quad y_{t}(x, 0)=-\pi^{2} \sin \pi x, \quad 0 \leq x \leq 1
$$

and with appropriate boundary conditions

$$
\left.\begin{array}{rl}
y(0, t)=0, \quad y(1, t) & =0, \quad t \geq 0, \\
y_{x x}(0, t)=0, \quad y_{x x}(1, t) & =0, \quad t \geq 0,
\end{array}\right\}
$$

The exact solution for this problem is $y(x, t)=e^{-\pi^{2} t} \sin \pi x$.

Upon discretization of the time variable, we obtain,

$$
\frac{y_{m+1}-2 y_{m}+y_{m-1}}{(\Delta t)^{2}}-\frac{d^{4} y_{m}}{d x^{4}}=g_{m}, \quad 0 \leq x \leq 1, \quad m=1, \ldots M-1
$$

where $\Delta t, t_{m}, m=0,1, \ldots, M, y, y_{m}(x) \approx y\left(x, t_{m}\right), g$ and $g_{m}$ are as expressed in example 1 , which is expressed in the form

$$
y^{(4)}=f\left(x, y, y^{\prime}, y^{\prime \prime}, y^{\prime \prime \prime}\right)=A y+g
$$

$A$ is as expressed in problem 5 and $g_{m}=0$.

Table 6: Error of methods for Problem 5: $\mathrm{h}=0.05$

\begin{tabular}{lll}
\hline$x y_{\text {app }}$ & $y_{\text {ex }}$ & Er \\
\hline 0.10 .3090 & 0.3090 & $1.5415 \times 10^{-13}$ \\
0.20 .5878 & 0.5878 & $5.5454 \times 10^{-13}$ \\
0.30 .8090 & 0.8090 & $8.1254 \times 10^{-13}$ \\
0.40 .9511 & 0.9511 & $1.3251 \times 10^{-13}$ \\
0.50 .9999 & 0.9999 & $8.4451 \times 10^{-13}$ \\
0.60 .9511 & 0.9511 & $8.1115 \times 10^{-13}$ \\
0.70 .8090 & 0.8090 & $9.8157 \times 10^{-13}$ \\
0.80 .5878 & 0.5878 & $8.3254 \times 10^{-13}$ \\
0.90 .3090 & 0.3090 & $2.2124 \times 10^{-13}$ \\
$1.01 .2246 \times 10^{-16} 1.2246 \times 10^{-16} 0$ \\
\hline
\end{tabular}

Table 7: Mximum error of methods for Problem 5, with $h=0.05$

\begin{tabular}{crccccc}
\hline Method & $k$ & $x=0.1$ & $x=0.2$ & $x=0.3$ & $x=0.4$ & $x=0.5$ \\
\hline SBUI & 10 & $6.56 \mathrm{E}-12$ & $6.54 \mathrm{E}-12$ & $6.65 \mathrm{E}-10$ & $6.12 \mathrm{E}-10$ & $5.54 \mathrm{E}-10$ \\
& 16 & $6.54 \mathrm{E}-13$ & $1.57 \mathrm{E}-11$ & $5.15 \mathrm{E}-11$ & $1.32 \mathrm{E}-11$ & $5.51 \mathrm{E}-11$ \\
Method in 24 & 10 & $2.91 \mathrm{E}-6$ & $1.73 \mathrm{E}-6$ & $1.60 \mathrm{E}-6$ & $2.23 \mathrm{E}-6$ & $2.60 \mathrm{E}-7$ \\
& 16 & $4.47 \mathrm{E}-7$ & $2.66 \mathrm{E}-7$ & $1.37 \mathrm{E}-7$ & $1.55 \mathrm{E}-7$ & $1.57 \mathrm{E}-7$
\end{tabular}


A six-sten block unification integrator for BVPs

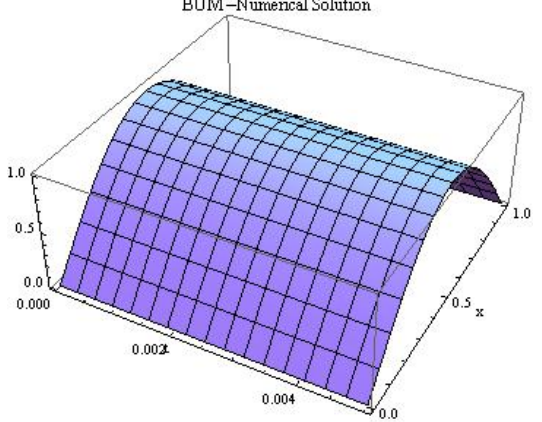

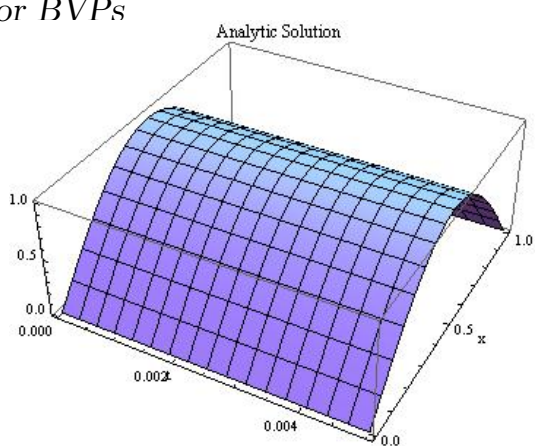

Fig. 1: Graphical representation for 32 showing surface plots for the numerical solution using SBUI, the analytic solution and the error $(e)$, for example 5 , where $e=\left|y_{n, m}-y\left(x_{n}, t_{m}\right)\right|$

Table 8: Error of methods for Problem 6, $\mathrm{h}=0.05$.

\begin{tabular}{lll}
\hline \multicolumn{1}{c}{$y_{\text {app }}$} & $y_{\text {ex }}$ & Er \\
\hline 0.10 .2913 & 0.2912 & $8.6565 \times 10^{-6}$ \\
0.20 .5540 & 0.5540 & $4.5455 \times 10^{-6}$ \\
0.30 .7625 & 0.7625 & $6.5451 \times 10^{-6}$ \\
0.40 .8964 & 0.8964 & $9.2555 \times 10^{-6}$ \\
0.50 .9425 & 0.9425 & $1.7545 \times 10^{-6}$ \\
0.60 .8964 & 0.8964 & $1.8458 \times 10^{-6}$ \\
0.70 .7625 & 0.7625 & $7.1111 \times 10^{-6}$ \\
0.80 .5540 & 0.5540 & $3.4545 \times 10^{-6}$ \\
0.90 .2912 & 0.2912 & $1.4545 \times 10^{-6}$ \\
$1.01 .1542 \times 10^{-16} 5.8454 \times 10^{-16} 0$ \\
\hline
\end{tabular}

Table 9: Maximum error of methods for Problem 6

\begin{tabular}{crccccccc}
\hline Method & Time & \multicolumn{2}{c}{ Parameter } & $x=0.1$ & $x=0.2$ & $x=0.3$ & $x=0.4$ & $x=0.5$ \\
\hline SBUI & $t=0.02$ & $h=0.05, \quad k=0.005$ & $2.10 \mathrm{E}-7$ & $4.00 \mathrm{E}-9$ & $5.51 \mathrm{E}-9$ & $6.48 \mathrm{E}-9$ & 6.81 & $\mathrm{E}-9$ \\
& $t=0.05$ & $h=0.05, \quad k=0.005$ & $1.12 \mathrm{E}-8$ & $2.14 \mathrm{E}-8$ & $2.94 \mathrm{E}-8$ & $3.46 \mathrm{E}-8$ & 3.64 & $\mathrm{E}-8$ \\
Method in 20] and $t=0.02$ & $n=91, \quad k=0.005$ & $3.34 \mathrm{E}-5$ & $6.35 \mathrm{E}-5$ & $8.74 \mathrm{E}-5$ & $1.03 \mathrm{E}-4$ & 1.08 & $\mathrm{E}-4$ \\
& $t=0.05$ & $n=91, \quad k=0.005$ & $3.33 \mathrm{E}-5$ & $6.34 \mathrm{E}-5$ & $8.73 \mathrm{E}-5$ & $1.03 \mathrm{E}-4$ & 1.08 & $\mathrm{E}-4$ \\
\hline
\end{tabular}
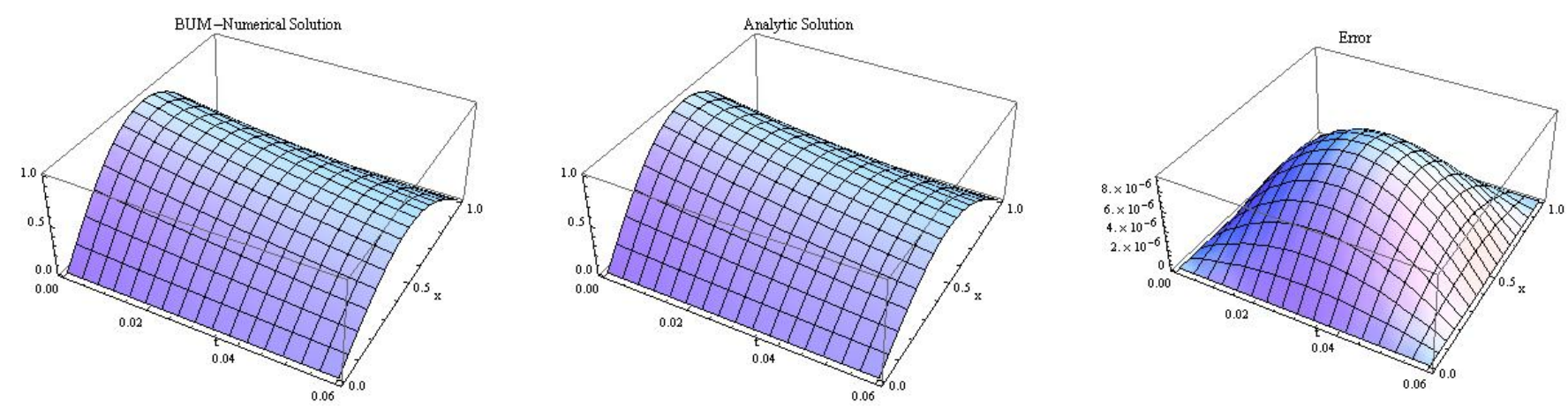

Fig. 2: Graphical representation for (34) showing surface plots for the numerical solution using SBUI, the analytic solution and the error $(e)$, for example 6 , where $e=\left|y_{n, m}-y\left(x_{n}, t_{m}\right)\right|$

Table 4 show the approximate and exact numerical solutions to problem 4 . On the other hand, Table 5 shows the maximum absolute error obtained in methods in 28, [13] and 29, as compared with the SBUI derived in the this work. For small values of $N=4,8,12$, it shows the efficiency in terms of the accuracy (as seen in the errors)

Table 6 shows the absolute errors obtained for problem 5. In Table 7 at different values of $k$ and points of $x$, shows the maximum absolute errors obtained, in comparison with the SBUI and the method in [24]. This shows the superiority of the 
SBUI. Figure 1 shows the graphical representation with surface plot for the numerical solution, analytical solution and residue (errors), for problem 5 .

Table 8 shows the absolute errors obtained in problem 6 . In Table 9 for different values of the parameters $k, t, h$ and $n$ and at different points of $x$, for $t=0.02$ and $t=0.05$ respectively, shows the maximum absolute errors obtained when the SBUI was compared with the method in 20. This shows that the SBUI performed better. Figure 2 shows the graphical representation with surface plot for the numerical solution, analytical solution and residue (errors), for problem 6 .

\section{Conclusion}

Six-step Block Unification Integrator, SBUI, have been derived from unifying linear multistep Methods (LMMs), and applied to solve BVPs and parabolic PDEs with appropriate boundary conditions, that have applications in mathematical physics. This method was applied directly without first reducing ODEs to an equivalent first order system. Standard numerical examples in literature were used to show the efficiency in terms of the techniques, accuracy in terms of the errors obtained, of the derived method when compared to other methods. It was also observed that the proposed method compare favourably and superior to existing methods in the literature cited.

\section{References}

[1] F. A. Costabile, A. Napoli. Collocation for high order differential equations with two-points Hermite boundary conditions. J. Appl. Numerical Mathematics 87 (2015) 157-167 https://doi.org/10.1016/j.apnum.2014.09.008

[2] M. K. Jain and T. Aziz, Cubic spline solution of two-point boundary Value with significant first derivatives, Comp.Meth. Appl.Mech. Eng., 39 (1983), pp. 83-91 https://doi.org/10.1016/0045-7825(83)90075-0

[3] S. N. Jator, Numerical Integrators For Fourth Order Initial And Boundary Value Problems. Inter. J. Pure and Appl. Math. 47, (4), (2008), 563-576

[4] H. B. Keller, Numerical methods for two-point boundary value problems, Blaisdell, Waltham, Mass.; 184 pp. QA372.K42 (1968).

[5] D. Krajcinivic, Sandwich Beam Analysis, Journal of Applied Mechanics $39 \quad(1972) \quad 773-778$. https://doi.org/10.1115/1.3422787

[6] X.Y. Li., B.Y. Wu, A Novel Method For Nonlinear Singular Fourth Order Four-Point Boundary Value Problems. Comp. Math Appl 62 (2011) 27-31. https://doi.org/10.1016/j.camwa.2011.04.029

[7] A.A. Salama, Higher-Order Method For Solving Free Boundary Value Problems, Numerical Heat Transfer, Part B: Fundamentals:An International Journal of Computation and Methodology 45 (2004) 385-394. https://doi.org/10.1080/10407790490278002

[8] D.X. Ma, X.Z. Yang, Upper and lower solution method for fourth-order four-point boundary value problems, Journal of Computational and Applied Mathematics 223 (2009) 543-551. https://doi.org/10.1016/j.cam.2007.10.051

[9] Q. Zhang, S.H. Chen, J.H. Lv, Upper and lower solution method for fourth-order four-point boundary value problems, Journal of Computational and Applied Mathematics 196 (2006) 387-393. https://doi.org/10.1016/j.cam.2005.09.007

[10] Y.L. Zhong, S.H. Chen, C.P. Wang, Existence results for a fourth-order ordinary differential equation with a four-point boundary condition, Applied Mathematics Letters 21 (2008) 465-470 https://doi.org/10.1016/j.aml.2007.03.029

[11] T. A. Biala, S.N. Jator, R.B. Adeniyi, Numerical approximations of second order PDEs by boundary value methods and the method of lines, Springer Plus (2015) 4:588 https://doi.org/10.1007/s13370-016-0458-4

[12] M. Devakar, D. Sreenivasu, B. Shankar, Analytical solutions of couple stress fluid flows with slip boundary conditions, Alexandria Engineering Journal (2014) 53, 723-730 https://doi.org/10.1016/j.aej.2014.06.005

[13] J. Duan, R. Rach, A new modification of the Adomian decomposition method for solving boundary value problems for higher order nonlinear differential equations, Appl. Math. Comput. (2011) 218, 4090-4118. https://doi.org/10.1016/j.amc.2011.09.037 
[14] K. Hussain, F. Ismail and N. Senua, Runge-Kutta Type Methods for Directly Solving Special Fourth-Order Ordinary Differential Equations: Mathematical Problems in Engineering (2015), Article ID 893763. https://doi.org/10.1155/2015/893763

[15] J.D. Lambert, Computational Methods in Ordinary Differential Equations,John Wiley, New York (1973).

[16] K. Hussain, F. Ismail and N. Senua, Solving directly special fourth-order ordinary differential equations using RungeKutta type method. Journal of Computational and Applied Mathematics (2016) 306, 179-199. https://doi.org/10.1016/j.cam.2016.04.002

[17] S.N. Jator, Block Unification Scheme for Elliptic, Telegraph, and Sine-Gordon Partial Differential Equations. American Journal of Computational Mathematics (2015) 5, 175-185. http://dx.doi.org/10.4236/ajcm.2015.52014.

[18] S.N. Jator, V. Manathunga, Block Nystrm type integrator for Bratus equation: Journal of Computational and Applied Mathematics (2018) 327, 341-349 https://doi.org/10.1016/j.cam.2017.06.025 .

[19] R. C. Mittal, R. K. Jain, B-splines methods with redefined basis functions for solving fourth order parabolic partial differential equations, Appl. Math. and Comp.(2011) 217, 9741-9755. https://doi.org/10.1016/j.amc.2011.04.061

[20] R. K. Mohanty, D. Kaur, High accuracy implicit variable mesh methods for numerical study of special types of fourth order nonlinear parabolic equations, Appl. Math. and Comp. (2016) 273, 678-696. https://doi.org/10.1016/j.amc.2015.10.036

[21] R. K. Mohanty, D. Kaur, Numerov type variable mesh approximations for 1D unsteady quasi-linear biharmonic problem: application to Kuramoto-Sivashinsky equation, Numerical Algorithms , (2017) 74,427-459. https://doi.org/10.1007/s11075-016-0154-3

[22] M.A Noor, S. T. Mohyud-Din, An efficient method for fourth order boundary value problems, Comput. Math. Appl. (2007) 54, 1101-1111.

[23] F.F. Ngwane and S.N. Jator, A Trigonometrically Fitted Block Method for Solving Oscillatory Second-Order Initial Value Problems and Hamiltonian Systems, Inter. J. of Diff. Eqn. (2017), Article ID 9293530, 14 pages. https://doi.org/10.1155/2017/9293530

[24] J. Rashidinia and R. Mohammadi, Sextic spline solution of variable coefficient fourth-order parabolic equations, Int. J. of Comp. Math. (2010) 87 (15) 3443-3454

https://doi.org/10.1080/00207160903085820

[25] P. Saucez, A. Vande Wouwer, W.E. Schiesser, P. Zegeling, Method of lines study of nonlinear dispersive waves, J of Computational and App Math . (2014) 168, 413-423. https://doi.org/10.1016/j.cam.2003.12.012

[26] Siddiqi S. S. and Akram G. (2008), Quintic Spline Solutions Of Fourth Order Boundary-Value Problems, Inter. Journal of Numerical Analysis And Modeling, 5, (1), 101-111.

[27] Siddiqi S. S., Arshed S. (2014), Quintic B-spline for the numerical solution of the good Boussinesq equation, J. Egypt. Math. Soc. 22, 209-213. https://doi.org/10.1016/j.joems.2013.06.015

[28] Tatari M., Dehghan M. (2006), The use of the Adomian decomposition method for solving multipoint boundary value problems, Phys. Scr. 73, 672-676. https://doi.org/10.1088/0031-8949/73/6/023

[29] Xu. M. Q., Lin Y. Z., Wang Y. H., (2016), A new algorithm for nonlinear fourth order multi-point boundary value problems, Applied Mathematics and Computation 274, 163-168 https://doi.org/10.1016/j.amc.2015.10.041 